\title{
Evaluation of BER for AWGN, Rayleigh and Rician Fading Channels under Various Modulation Schemes
}

\author{
A. Sudhir Babu \\ Associate Professor, Department of CSE, \\ PVP Siddhartha Institute of Technology, \\ Vijayawada, India
}

\author{
Dr. K.V Sambasiva Rao \\ Professor and Principal \\ MVR College of Engineering and Technology, \\ Paritala, Vijayawada, India
}

\begin{abstract}
Several transmission modes are defined in IEEE $802.11 \mathrm{a} / \mathrm{b} / \mathrm{g}$ WLAN standards. A very few transmission modes are considering for IEEE $802.11 \mathrm{a} / \mathrm{b} / \mathrm{g}$ in physical layer parameters and wireless channel characteristics. In this paper, we evaluated the performance of available transmission modes in IEEE 802.11b [1]. However, the performance analysis can be done straightforward using the evaluation of IEEE 802.11b. The performance of transmission modes are evaluated by calculating the probability of Bit Error Rate (BER) versus the Signal Noise Ratio (SNR) under the frequently used three wireless channel models (AWGN, Rayleigh and Rician) [2]. We consider the data modulation and data rate to analyze the performance that is BER vs. SNR. We also consider multipath received signals. The simulation results had shown the performance of transmission modes under different channel models and the number of antennas. Based on simulation results, we observed that some transmission modes are not efficient in IEEE 802.11b. The evaluation of performance confirms the increase in the coverage area of the physical layer in the $802.11 \mathrm{~b}$ WLAN devices.
\end{abstract}

\section{General Terms}

Digital Modulation, Fading, BER (Bit Error Ratio), SNR (Signal to Noise Ratio)

\section{Keywords}

AWGN, Rayleigh, Rician

\section{INTRODUCTION}

It is important to evaluate the performance of wireless devices by considering the transmission characteristics, wireless channel parameters and device structure. The performance of data transmission over wireless channels is well captured by observing their BER, which is a function of SNR [3] at the receiver. In wireless channels, several models have been proposed and investigated to calculate SNR. All the models are a function of the distance between the sender and the receiver, the path loss exponent and the channel gain. Several probability distributed functions are available to model a time-variant parameter i.e. channel gain. We describe the three important and frequently used distributions. Those are AWGN, Rayleigh and Rician models. The signal is detected and decoded by employing several replicas of the received signal. So, we consider multilink receiver structure. The PHY layer transmission mode consists of a specific set of modulation, binary convolution coding and data rate. According to IEEE 802.11 standard [1], each device should use a wireless transmission technique among OFDM (Orthogonal Frequency Division Multiplexing), DSSS (Direct Sequence Spread Spectrum), FHSS (Frequency Hopping Spread
Spectrum) and IR (InfraRed). In our evaluation, we consider OFDM and DSSS. For binary convolution coding, the receiver uses a Viterbi decoding, which is recommended by the IEEE standard.

\section{WIRELESS CHANNEL MODELING}

Wireless communication is one of the most active areas of technology development and has become an ever-more important and prominent part of everyday life. Simulation of wireless channels accurately is very important for the design and performance evaluation of wireless communication systems and components. Fading or loss of signals is a very important phenomenon that related to the Wireless Communications Field. That leads us to the fading models which try to describe the fading patterns in different environments and conditions. Although no model can 'perfectly' describe an environment, they strive to obtain as much precision as possible. The better a model can describe a fading environment, the better can it be compensated with other signals, so that, on the receiving end, the signal is error free or at least close to being error free. This would mean higher clarity of voice and higher accuracy of data transmitted over wireless medium. An important issue is in wireless application development is the selection of fading models. A comparative analysis of DPSK and QAM [4] will also provide knowledge base which helps for application development in real-world.

\subsection{Fading and Multipath}

Fading refers to the distortion that a carrier-modulated telecommunication signal experiences over certain propagation media. In wireless systems, fading is due to multipath propagation and is sometimes referred to as multipath induced fading. To understand fading, it is essential to understand multipath. In wireless telecommunications, multipath is the propagation phenomenon that results in radio signals' reaching the receiving antenna by two or more paths. Causes of multipath include atmospheric ducting, ionospheric reflection and refraction, and reflection from terrestrial objects, such as mountains and buildings. The effects of multipath include constructive and destructive interference, and phase shifting of the signal. This distortion of signals caused by multipath is known as fading. In other words it can be said that in the real world, multipath occurs when there is more than one path available for radio signal propagation. The phenomenon of reflection, diffraction and scattering all give rise to additional radio propagation paths beyond the direct optical LOS[2] (Line of Sight) path between the radio transmitter and receiver. 


\subsection{Fading Channels}

A Fading Channel is known as communications channel which has to face different fading phenomenon's, during signal transmission. In real world environment, the radio propagation effects combine together and multipath is generated by these fading channels. Due to multiple signal propagation paths, multiple signals will be received by receiver and the actual received signal level is the vector sum of the all signals. These signals incident from any direction or angle of arrival. In multipath, some signals aid the direct path and some others subtract it.

\subsection{Causes of Fading}

Fading is caused by different physical phenomenon:

\subsubsection{Doppler Shift:}

When a mobile is moving at a constant velocity $v$ along a path, $v_{s}$ is the velocity of the source, $f$ 'is the observed frequency and $f$ is the emitted frequency. All these terms will be related by the following equation:

$$
f^{\prime}=\left(\frac{v}{v \pm v s}\right) f
$$

From the above equation, that the detected frequency increases for objects moving towards the observer and decreases when the source moves away. This phenomena is known as the Doppler Effect [4].

\subsubsection{Reflection:}

When a propagating electromagnetic wave impinges on object which has generated large dimensions wave length, when compared to wavelength of the propagating wave, then Reflection will occurred. Actually we know that if the plane wave is incident on a perfect dielectric, part of the energy is transmitted and part of the energy is reflected back into the medium. If the medium is a perfect conductor, all the energy is reflected back. Reflections occur from the surface of the earth and from buildings and walls. In practice, not only metallic materials cause reflections, but dielectrics also cause this phenomenon [5].

\subsubsection{Diffraction:}

The sharp irregularities (edges) of a surface between transmitter and receiver and obstructs the radio path then diffraction will occurred. The bending waves around the obstacle, even when a Line of Sight does not exist between transmitter and receiver the secondary waves will be spread over the space. Diffraction looks like a reflection at high frequencies depends on the amplitude, phase and polarization of the incident wave and geometry of the object at the point of diffraction.

\subsubsection{Scattering:}

The wave travels through the medium consists of smaller dimension objects compared to the wavelength and having larger volumes of obstacles per unit volume, then scattering will occurred. Due to rough surfaces, small objects and irregularities in the channel scattered waves are produced. In practice, in mobile communications, electrical poles and street signs etc. induces scattering [6] in communication.

\subsection{Types of Fading}

According to the effect of multipath, there are two types of fading

a). Large Scale Fading, In this type of fading, the received signal power varies gradually due to signal attenuation determined by the geometry of the path profile.

b). Small Scale Fading If the signal moves over a distance in the order of wavelength, in small scale fading leads to rapid fluctuation of the phase and amplitude of the signal.

Flat Fading If the bandwidth of the mobile channel is greater than the bandwidth of the transmitted channel, it causes flat fading. Flat fading is one in which all frequency components of a received radio signal vary in the same proportion simultaneously.

There are two types of fading according to the effect of Doppler Spread.

a). Slow fading When the coherence time of the channel is large relative to the delay constraint of the channel then slow fading will occurred. The amplitude and phase change imposed by the channel can be considered roughly constant over the period of use. The events such as shadowing, where a large obstruction such as a hill or large building obscures the main signal path between the transmitter and the receiver, causes the slow fading..

b). Fast fading When the coherence time of the channel is small relative to the delay constraint of the channel causes the fast fading. The amplitude and phase change imposed by the channel varies considerably over the period of use.

\subsubsection{Types of small scale fading}

There are many models that describe the phenomenon of small scale fading. Out of these models, Rayleigh fading, Ricean fading and Nakagami fading models are most widely used.

a). Rayleigh fading model: The Rayleigh fading is primarily caused by multipath reception [6]. Rayleigh fading is a statistical model for the effect of a propagation environment on a radio signal. It is a reasonable model for troposphere and ionospheres' signal propagation as well as the effect of heavily built-up urban environments on radio signals. Rayleigh fading [7] is most applicable when there is no line of sight between the transmitter and receiver.

b). Ricean fading model: The Ricean fading model [6] is similar to the Rayleigh fading model, except that in Ricean fading, a strong dominant component is present. This dominant component is a stationary (non fading) signal and is commonly known as the LOS (Line of Sight Component).

c). Additive White Gaussian Noise Model: The simplest radio environment in which a wireless communications system or a local positioning system or proximity detector based on Timeof-flight will have to operate is the Additive-White Gaussian Noise (AWGN) [4] environment. Additive white Gaussian noise (AWGN) is the commonly used to transmit signal while signals travel from the channel and simulate background noise of channel. The mathematical expression in received signal

$\mathrm{r}(\mathrm{t})=\mathrm{s}(\mathrm{t})+\mathrm{n}(\mathrm{t})$ that passed through the AWGN channel where $\mathrm{s}(\mathrm{t})$ is transmitted signal and $\mathrm{n}(\mathrm{t})$ is background noise. 
An AWGN channel adds white Gaussian noise to the signal that passes through it. It is the basic communication channel model and used as a standard channel model. The transmitted signal gets disturbed by a simple additive white Gaussian noise process.

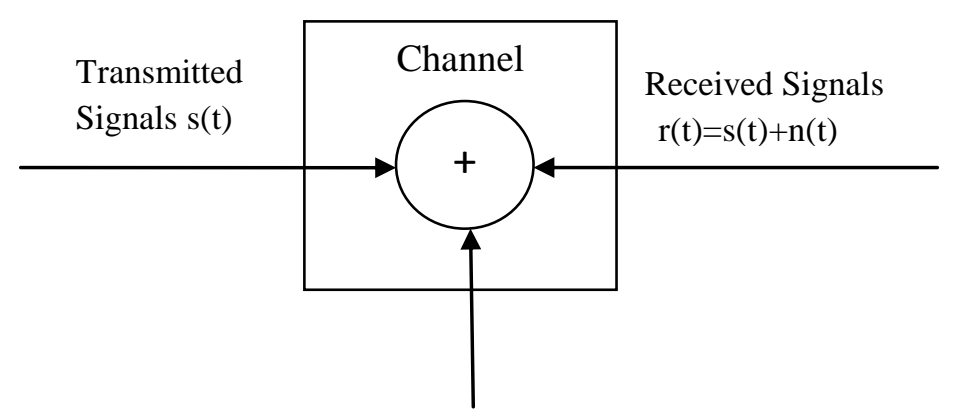

Figure 1. Block Diagram of AWGN Channel model

\section{MODULATION}

One way to communicate a message signal whose frequency spectrum does not fall within that fixed frequency range, or one that is unsuitable for the channel, is to change a transmittable signal according to the information in the message signal. This alteration is called modulation, and it is the modulated signal that is transmitted. The receiver then recovers the original signal through a process called demodulation.

Modulation techniques are expected to have three positive properties:

- Good Bit Error Rate Performance: Modulation schemes should achieve low bit error rate in the presence of fading, Doppler spread, interference and thermal noise.

- Power Efficiency: Power limitation is one of the critical design challenges in portable and mobile applications. Nonlinear amplifiers are usually used to increase power efficiency. However, nonlinearity may degrade the bit error rate performance of some modulation schemes. Constant envelope modulation techniques are used to prevent the re growth of spectral side lobes during nonlinear amplification

- Spectral Efficiency: The modulated signals power spectral density should have a narrow main lobe and fast roll-off of side lobes. Spectral efficiency is measured in units of $\mathrm{bit} / \mathrm{sec} / \mathrm{Hz}$.

\subsection{Digital Modulation}

Digital modulation schemes transform digital signals into waveform that are compatible with the nature of the communications channel. One category uses a constant amplitude carrier and the other carries the information in phase or frequency variations (FSK, PSK). A major transition from the simple amplitude modulation (AM) and frequency modulation (FM) to digital techniques such as Quadrature Phase Shift Keying (QPSK), Frequency Shift Keying (FSK), Minimum Shift Keying (MSK) and Quadrate Amplitude Modulation (QAM).

\subsubsection{6-Quadrature Amplitude Modulation:}

QAM is the encoding of the information into a carrier wave by variation of the amplitude of both the carrier wave and a 'quadrature' carrier that is $90^{\circ}$ out of phase with the main carrier in accordance with two input signals. That is, the amplitude and the phase of the carrier wave are simultaneously changed according to the information you want to transmit.

In 16-in 16-state Quadrature Amplitude Modulation (16-QAM), there are four I values and four Q values. This results in a total of 16 possible states for the signal. It can transition from any state to any other state at every symbol time. Since $16=2^{4}$, four bits per symbol can be sent. This consists of two bits for I and two bits for Q. The symbol rate is one fourth of the bit rate. So this modulation format produces a more spectrally efficient transmission. It is more efficient than BPSK, QPSK or 8PSK. Note that QPSK is the same as 4-QAM [8].

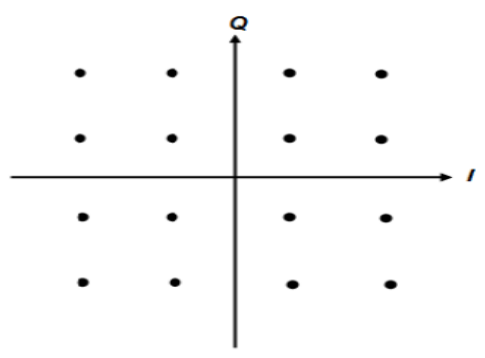

Figure 2. 16-QAM Constellation

\subsubsection{4-Quadrature Amplitude Modulation:} 64-QAM is same as 16-QAM except it is 64 possible signal combinations with each symbol represent six bits $\left(2^{6}=64\right)$. 64QAM [8] is a complex modulation technique but gives high efficiency. This digital frequency modulation technique is primarily used for sending data downstream over a coaxial cable network. 64QAM is very efficient, supporting up to $28-\mathrm{mbps}$ peak transfer rates over a single 6-MHz channel. But 64QAM's susceptibility to interfering signals makes it ill suited to noisy upstream transmissions.

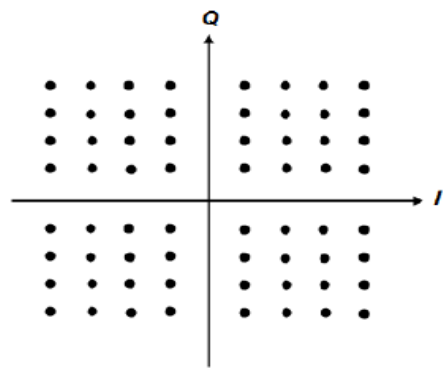

Figure 3. Constellation Diagtam for 64-QAM

\subsubsection{Differential Phase Shift Keying:}

Differential phase shift keying (DPSK) [8], a common form of phase modulation conveys data by changing the phase of carrier wave. In Phase shift keying, High state contains only one cycle but DPSK contains one and half cycle. 
Differential Shift Keying is a modulation technique that codes information by using the phase difference between two neighboring symbols. In the transmitter, each symbol is modulated relative to the previous symbol and modulating signal, for instance in BPSK 0 represents no change and 1 represents +180 degrees. In the receiver, the current symbol is demodulated using the previous symbol as a reference. The previous symbol serves as an estimate of the channel. A no change condition causes the modulated signal to remain to remain at the same 0 or 1 state of the previous symbol. Differential modulation is theoretically $3 \mathrm{~dB}$ poorer than coherent. This is because the differential system has 2 sources of error: a corrupted symbol, and a corrupted reference.

In DPSK, the transmitter, each symbol is modulated relative to the phase of the immediately preceding signal element and the data being transmitted. In this paper, we choose 16-DPSK and 64-DPSK schemes to analyze BER and SNR in different fading channels.

\subsection{Bit Error Rate (BER)}

The BER, or quality of the digital link, is calculated from the number of bits received in error divided by the number of bits transmitted.

$\mathrm{BER}=($ Bits in Error $) /($ Total bits received $)$.

In digital transmission, the number of bit errors is the number of received bits of a data stream over a communication channel that has been altered due to noise, interference, distortion or bit synchronization errors. The BER is the number of bit errors divided by the total number of transferred bits during a particular time interval. BER is a unit less performance measure, often expressed as a percentage.

IEEE 802.11 standard has ability to sense the bit error rate (BER) of its link and implemented modulation to data rate and exchange to Forward Error Correction (FEC), which is used to set the BER as low error rate for data applications. BER measurement is the number of bit error or destroys within a second during transmitting from source to destination.

Noise affects the BER performance. Quantization errors also reduce BER performance, through incorrect or ambiguous reconstruction of the digital waveform. The accuracy of the analog modulation process and the effects of the filtering on signal and noise bandwidth also effect quantization errors.

BER can also be defined in terms of the probability of error POE) [9] and represented in Eq. (1).

$$
\mathrm{POE}=\frac{1}{2}(1-e r f) \sqrt{\frac{E b}{N 0}}
$$

where erf is the error function, $E_{b}$ is the energy in one bit and $\mathrm{N}_{0}$ is the noise power spectral density (noise power in a $1 \mathrm{~Hz}$ bandwidth). The error function is different for the each of the various modulation methods. The POE is a proportional to $\mathrm{E}_{\mathrm{b}} / \mathrm{N}_{0}$, which is a form of signal-to-noise ratio. The energy per bit, $\mathrm{E}_{\mathrm{b}}$, can be determined by dividing the carrier power by the bit rate. As an energy measure, $\mathrm{E}_{\mathrm{b}}$ has the unit of joules. $\mathrm{N}_{0}$ is in power that is joules per second, so, $\mathrm{E}_{\mathrm{b}} / \mathrm{N}_{0}$ is a dimensionless term, or is a numerical ratio.

\subsection{Signal to Noise Ratio (SNR)}

SNR is the ratio of the received signal strength over the noise strength in the frequency range of the operation. It is an important parameter of the physical layer of Local Area Wireless Network (LAWN). Noise strength, in general, can include the noise in the environment and other unwanted signals (interference). BER is inversely related to SNR, that is high BER causes low SNR. High BER causes increases packet loss, increase in delay and decreases throughput. The exact relation between the SNR and the BER is not easy to determine in the multi channel environment. Signal to noise ratio (SNR) is an indicator commonly used to evaluate the quality of a communication link and measured in decibels and represented by Eq. (2).

SNR $=10 \log _{10}($ Signal Power $/$ Noise Power $)$ dB. --- (2)

\section{$3.4 E_{b} / N_{0}$ (Energy per bit to Noise power spectral density ratio)}

$\mathbf{E}_{\mathbf{b}} / \mathbf{N}_{\mathbf{0}}$ is an important parameter in digital communication or data transmission. It is a normalized signalto-noise ratio (SNR) measure, also known as the "SNR per bit". It is especially useful when comparing the bit error rate (BER) performance of different digital modulation schemes without taking bandwidth into account. $\mathrm{E}_{\mathrm{b}} / \mathrm{N}_{0}$ is equal to the $\mathrm{SNR}$ divided by the "gross" link spectral efficiency in (bit/s)/Hz, where the bits in this context are transmitted data bits, inclusive of error correction information and other protocol overhead. When forward error correction (FEC) is being discussed, $\mathrm{E}_{\mathrm{b}} / \mathrm{N}_{0}$ is routinely used to refer to the energy per information bit (i.e. the energy per bit net of FEC overhead bits); in this context, $E_{\mathrm{s}} / \mathrm{N}_{0}$ is generally used to relate actual transmitted power to noise.

\section{SIMULATION RESULTS}

In this paper, one of the important topic in wireless communications, that is the concept of fading is demonstrated by the approach available in MATLAB. In this section, the results obtained from the MATLAB simulations [10] are discussed. It is necessary to explore what happens to the signal as it travels from the transmitter to the receiver. Then it is very easy to understand the concepts in wireless communications. As explained earlier, one of the important aspects of the path between the transmitter and receiver is the occurrence of fading. MATLAB provides a simple and easy way to demonstrate fading taking place in wireless systems. The $R_{f}$ (radio frequency) signals with appropriate statistical properties can readily be simulated. Statistical testing can subsequently be used to establish the validity of the fading models frequently used in wireless systems. The different fading models and MATLAB based simulation approaches will now be described.

Simulink is a graphical extension to MATLAB for the modeling and simulation of systems. In Simulink, systems are drawn on screen as block diagrams. Many elements of block diagrams are

available (such as transfer functions, summing junctions, etc.), as well as virtual input devices and output devices. Simulink is integrated with MATLAB and data can be easily transferred between the programs. 
Table 1: BER in AWGN, Rayleigh and Rician fading channels in 16-QAM modulation scheme

\begin{tabular}{|c|c|c|c|}
\hline $\mathrm{E}_{\mathrm{b}} / \mathrm{N}_{\mathrm{o}}$ & $\begin{array}{c}\text { BER in } \\
\text { AWGN }\end{array}$ & $\begin{array}{c}\text { BER in } \\
\text { Rayleigh }\end{array}$ & $\begin{array}{c}\text { BER in } \\
\text { Rician }\end{array}$ \\
\hline$-5: 0$ & 0.1064 & 0.1033 & 0.121 \\
\hline $0: 5$ & 0.0952 & 0.0944 & 0.0991 \\
\hline $5: 10$ & 0.0578 & 0.0608 & 0.0401 \\
\hline $10: 15$ & 0.0235 & 0.0275 & 0.001699 \\
\hline $15: 20$ & 0.0078 & 0.01 & $1.8399 \mathrm{E}-7$ \\
\hline $20: 25$ & 0.0025 & 0.0029 & $1.4 \mathrm{E}-19$ \\
\hline
\end{tabular}

Graph 1: Comparative Study of AWGN, Rayleigh and Rician in 16-QAM Modulation Scheme

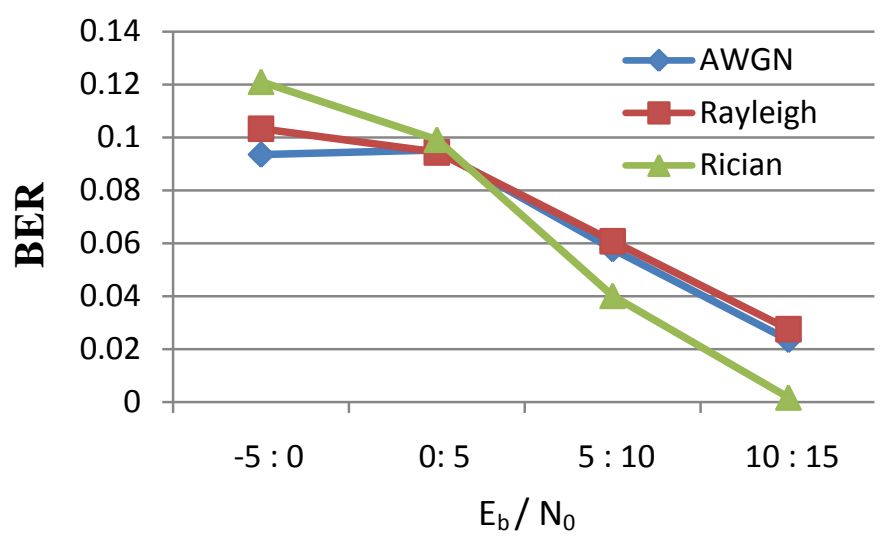

Table 2: BER in AWGN, Rayleigh and Rician fading channels in 64-QAM modulation scheme

\begin{tabular}{|c|c|c|c|}
\hline $\mathrm{E}_{\mathrm{b}} / \mathrm{N}_{\mathrm{o}}$ & $\begin{array}{c}\text { BER in } \\
\text { AWGN }\end{array}$ & $\begin{array}{c}\text { BER in } \\
\text { Rayleigh }\end{array}$ & BER in Rician \\
\hline$-5: 0$ & 0.0935 & 0.0905 & 0.1094 \\
\hline $0: 5$ & 0.0948 & 0.0935 & 0.0991 \\
\hline $5: 10$ & 0.0763 & 0.0769 & 0.0742 \\
\hline $10: 15$ & 0.0431 & 0.046 & 0.025728 \\
\hline $15: 20$ & 0.0168 & 0.02 & 0.000772 \\
\hline $20: 25$ & 0.0056 & 0.0072 & 0.0000000263 \\
\hline
\end{tabular}

Graph 2: Comparative Study of AWGN, Rayleigh and Rician in 64-QAM Modulation Scheme

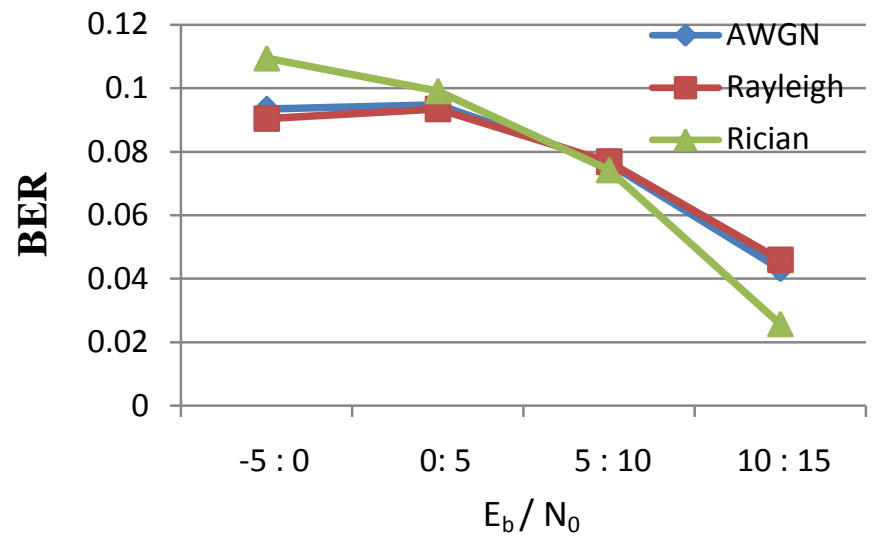

Table 3: BER in AWGN, Rayleigh and Rician fading channels in 16-DPSK modulation scheme

\begin{tabular}{|c|c|c|c|}
\hline $\mathrm{E}_{\mathrm{b}} / \mathrm{N}_{\mathrm{o}}$ & BER in AWGN & $\begin{array}{c}\text { BER in } \\
\text { Rayleigh }\end{array}$ & $\begin{array}{c}\text { BER in } \\
\text { Rician }\end{array}$ \\
\hline$-5: 0$ & 0.1212 & 0.0946 & 0.0986 \\
\hline $0: 5$ & 0.1102 & 0.101 & 0.1027 \\
\hline $5: 10$ & 0.0787 & 0.0861 & 0.0855 \\
\hline $10: 15$ & 0.0472 & 0.0587 & 0.0565 \\
\hline $15: 20$ & 0.006899 & 0.0293 & 0.0259 \\
\hline $20: 25$ & 0.000000220999 & 0.0114 & 0.0092 \\
\hline
\end{tabular}

Graph 3: Comparative Study of AWGN, Rayleigh and Rician in 16-DPSK Modulation Scheme

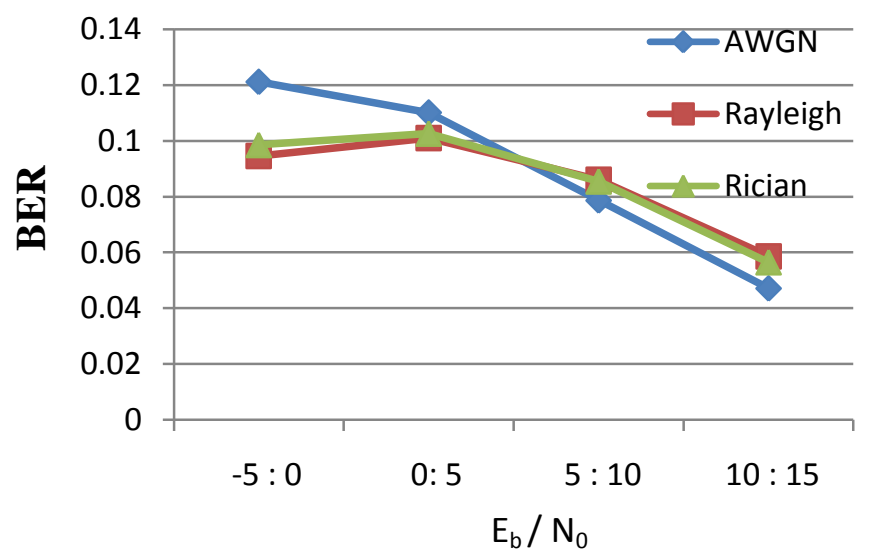


Table 4: BER in AWGN, Rayleigh and Rician fading channels in 64-DPSK modulation scheme

\begin{tabular}{|c|c|c|c|}
\hline $\mathrm{E}_{\mathrm{b}} / \mathrm{N}_{\mathrm{o}}$ & $\begin{array}{c}\text { BER in } \\
\text { AWGN }\end{array}$ & $\begin{array}{c}\text { BER in } \\
\text { Rayleigh }\end{array}$ & $\begin{array}{c}\text { BER in } \\
\text { Rician }\end{array}$ \\
\hline$-5: 0$ & 0.0798 & 0.0659 & 0.0681 \\
\hline $0: 5$ & 0.0696 & 0.0662 & 0.0737 \\
\hline $5: 10$ & 0.0746 & 0.0737 & 0.074 \\
\hline $10: 15$ & 0.0697 & 0.0689 & 0.044 \\
\hline $15: 20$ & 0.0521 & 0.0575 & 0.0386 \\
\hline $20: 25$ & 0.0328 & 0.0396 & 0.0113 \\
\hline
\end{tabular}

Graph 4: Comparative Study of AWGN, Rayleigh and Rician in 64-DPSK Modulation Scheme

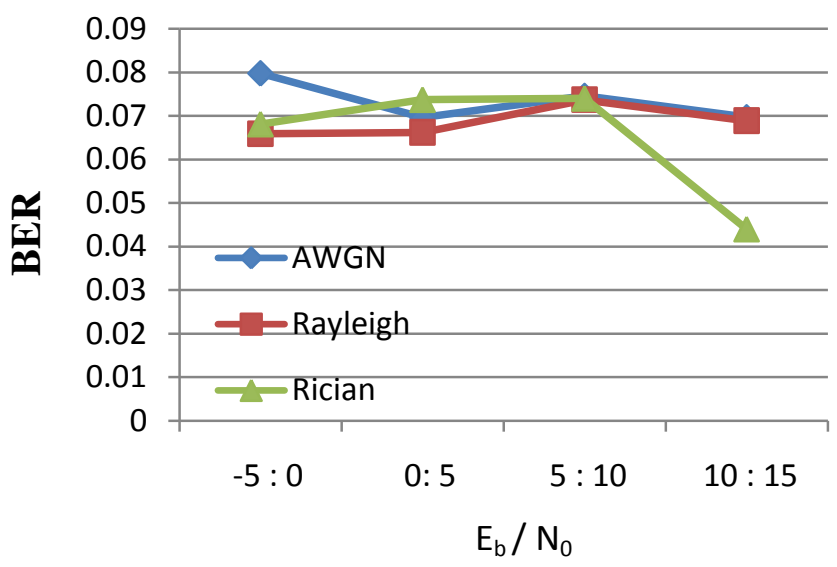

\section{CONCLUSION}

From the simulation results, The Bit Error Ratio of a digital communication system is an important figure of merit used to quantify the integrity of data transmitted through the system. By implementing the different modulation techniques, the criterion is comparison of the variation of BER for different SNR. It is observed that the BER is minimum for AWGN and maximum for RAYLEIGH and RICIAN. For RICIAN it is found that the BER is less than AWGN and RAYLEIGH except in case of 16-
DPSK. And it is observed that 16-QAM is performing better than 64-QAM. For higher values of $\mathrm{E}_{\mathrm{b}} / \mathrm{N}_{0}$, the BER is decreasing in all the fading channels for different modulation schemes.

\section{REFERENCES}

[1] IEEE standard for wireless LAN: Medium Access Control and Physical Layer Specification, P802.11, January 1999.

[2] Mohammaed Slim Alouini and Andrea J. Goldsmith “ Capacity of Rayleigh fading channels under different Adaptive Transmission and Diversity combining Techniques", IEEE Transactions on Vehicular Technology, Vol. 48, No. 4, July 1999.

[3]. Gary Breed, High Frequency Electronics, 2003 Summit, Technical Media LLC "Bit Error Rate: Fundamental Concepts and measurement issues".

[4] Fumiyaki Adachi, "error Rate Analysis of Differentially Encoded and detected 16-APSK under Rician fading", IEEE Transactions on Vehicular Technology, Vol. 45, No. 1, February 1996.

[5] Jiho Ryu, Jeong Keun Lee, Sung-Ju Lee and Taekyoung Kwon, "Revamping the IEEE 802.11a PHY Simulation Models”, MSWim' 08, October 27-31, 2008, Vancouver, BC, Canada.

[6] A. Alimohammad, S.F.Fard, B.F.Cockburn and C.Schlegal, "Compact Rayleigh and Rician fading simulation based on random walk processes", IET Communications, 2009, Vol. 3 , Issue 8, pp 1333-1342.

[7] Yahong Rosa Zheng and Chengshan Xiao, "Simulation models with correct statistical properties for Rayleigh fading channels", IEEE Transactions on communications, Vol. 51, No. 6, June 2003.

[8] "Quadrature Amplitude Modulation", digital Modulation Techniques" www.digitalmodulation.net/qam.html

[9] Lightwave Magazine, September 2004 article on "Explaining those BER testing mysteries".

[10] James E. Gilpy, Transcript International Inc., August 2003 , "Bit Error Rate Simulation using Matlab" 